\title{
HOMOGENIZATION OF MONOTONE SYSTEMS OF HAMILTON-JACOBI EQUATIONS
}

\author{
Fabio CAmilli ${ }^{1}$, Olivier Ley $^{2}$ And PaOla Loreti ${ }^{3}$
}

\begin{abstract}
In this paper we study homogenization for a class of monotone systems of first-order timedependent periodic Hamilton-Jacobi equations. We characterize the Hamiltonians of the limit problem by appropriate cell problems. Hence we show the uniform convergence of the solution of the oscillating systems to the bounded uniformly continuous solution of the homogenized system.
\end{abstract}

Mathematics Subject Classification. 35B27, 49L25, 35K45.

Received January 30, 2008.

Published online October 21, 2008.

\section{INTRODUCTION}

In this paper we study the behavior as $\varepsilon \rightarrow 0$ of the monotone system of Hamilton-Jacobi equations

$$
\begin{cases}\frac{\partial u_{i}^{\varepsilon}}{\partial t}+H_{i}\left(x, \frac{x}{\varepsilon}, u^{\varepsilon}, D u_{i}^{\varepsilon}\right)=0 & (x, t) \in \mathbb{R}^{N} \times(0, T], \\ u_{i}^{\varepsilon}(x, 0)=u_{0, i}(x) & x \in \mathbb{R}^{N}, i=1, \ldots, M,\end{cases}
$$

where the Hamiltonians $H_{i}(x, y, r, p), i=1, \ldots, M$, are periodic in $y$, coercive in $p$ and satisfy some uniform continuity properties, see (2.2). The $u_{0, i}$ 's are bounded uniformly continuous (BUC in short). The monotonicity condition, see (2.3), we assume for the system is a standard assumption to obtain a comparison principle for (1.1) (see $[10,12-14])$.

The main result of the paper, see Theorem 5.2, is the convergence of $u^{\varepsilon}$, as $\varepsilon \rightarrow 0$, to a BUC function $u=\left(u_{1}, \ldots, u_{M}\right)$ which solves in viscosity sense the homogenized system

$$
\begin{cases}\frac{\partial u_{i}}{\partial t}+\bar{H}_{i}\left(x, u, D u_{i}\right)=0 & (x, t) \in \mathbb{R}^{N} \times(0, T) \\ u_{i}(x, 0)=u_{0, i}(x) & x \in \mathbb{R}^{N}, i=1, \ldots, M\end{cases}
$$

The Hamiltonians $\bar{H}_{i}$ of the limit problem, the so-called effective Hamiltonians, are characterized by appropriate cell problems. The comparison principle for (1.2) which provides existence and uniqueness is not an immediate

\footnotetext{
Keywords and phrases. Systems of Hamilton-Jacobi equations, viscosity solutions, homogenization.

${ }^{1}$ Dip. di Matematica Pura e Applicata, Univ. dell'Aquila, loc. Monteluco di Roio, 67040 l'Aquila, Italy. camilli@ing.univaq. it

${ }^{2}$ Université François-Rabelais, Tours; Laboratoire de Mathématiques et Physique Théorique, CNRS UMR 6083, Fédération de Recherche Denis Poisson (FR 2964); Faculté des Sciences et Techniques, Parc de Grandmont, 37200 Tours, France.

ley@lmpt.univ-tours.fr

3 Dip. di Metodi e Modelli Matematici per le Scienze Applicate, Facoltà di Ingegneria, Sapienza Università di Roma, via Scarpa 16, 00161 Roma, Italy. loreti@dmmm.uniroma1.it
} 
consequence of the comparison principle for (1.1) since the regularity properties we could prove for the effective Hamiltonians are weaker than those for the initial ones (compare (2.2) and (4.3)).

Homogenization of Hamilton-Jacobi equations in the framework of viscosity solution theory was firstly considered in the seminal paper by Lions et al. [16]. The proof of our homogenization result relies on an appropriate modification of the classical perturbed test function method. This technique was introduced in the framework of the viscosity solutions theory by Evans [11] for the case of a periodic equation. Then it has been adapted to many different homogenization problems, see e.g. $[1,7,8,15]$. For a complete account of the homogenization theory in the periodic case we refer to [1].

Concerning the homogenization of systems of Hamilton-Jacobi equations we refer to [11,17]. In these papers, homogenization of weakly coupled systems, i.e. systems with a linear coupling, was considered together with a penalization of the coupling term of order $\varepsilon^{-1}$. Because of the penalization, the limit problem is a single Hamilton-Jacobi equation and all the components of the solution of the perturbed system converge to the unique solution of this equation.

We consider the more general class of monotone systems, which in particular includes the weakly coupled ones. Moreover, since we do not penalize the coupling term, the homogenized problem is still a system of Hamilton-Jacobi equations and the perturbed test function method has to be adapted to this situation.

In Section 6, we discuss in more details the homogenization of the weakly coupled systems. In particular we show that the homogenized system is not necessarily weakly coupled but only monotone. For a particular 1-dimensional weakly coupled eikonal system, we give an explicit formula for the effective Hamiltonians.

The plan of the paper is the following.

In Section 2 we describe our assumptions and definitions. In Section 3 we study the system (1.1) for $\varepsilon>0$. In Section 4, we define the effective Hamiltonians and we study their properties. In Section 5 we prove the homogenization result. In Section 6 we study some examples and in particular the weakly coupled systems. Finally in the Appendix we prove a comparison theorem for (1.1).

Notation: We will use the following norm

$$
|f|_{\infty}=\operatorname{esssup}_{x \in \mathbb{R}^{N}}|f(x)|
$$

and $B_{k}(x, R)$ denotes the $k$-dimensional ball of center $x \in \mathbb{R}^{N}$ and radius $R>0$.

\section{Assumptions AND PRELiminary RESUlts}

We consider the monotone system of Hamilton-Jacobi equations

$$
\begin{cases}\frac{\partial u_{i}^{\varepsilon}}{\partial t}+H_{i}\left(x, \frac{x}{\varepsilon}, u^{\varepsilon}, D u_{i}^{\varepsilon}\right)=0 & (x, t) \in \mathbb{R}^{N} \times(0, T), i=1, \ldots, M, \\ u_{i}^{\varepsilon}(x, 0)=u_{0, i}(x) & x \in \mathbb{R}^{N},\end{cases}
$$

where $u^{\varepsilon}=\left(u_{1}^{\varepsilon}, \ldots, u_{M}^{\varepsilon}\right)$ and $u_{i}^{\varepsilon}$ is a real valued function defined in $\mathbb{R}^{N} \times[0, T]$. We assume that the Hamiltonians $H_{j}: \mathbb{R}^{N} \times \mathbb{R}^{N} \times \mathbb{R}^{M} \times \mathbb{R}^{N} \rightarrow \mathbb{R}, j=1, \ldots, M$, are continuous and satisfy the following assumptions:

(i) $H_{j}(x, y, r, p)$ is $\mathbb{Z}^{N}$-periodic in $y$ for any $(x, r, p)$;

(ii) $H_{j}(x, y, r, p)$ is coercive in $p$, i.e.

$\lim _{|p| \rightarrow+\infty} H_{j}(x, y, r, p)=+\infty$ uniformly in $(x, y, r)$

(iii) for all $R>0, H_{j} \in B U C\left(\mathbb{R}^{N} \times \mathbb{R}^{N} \times[-R, R]^{M} \times B_{N}(0, R)\right.$ );

(iv) there exists a modulus of continuity $\omega$ s.t.

$$
\begin{aligned}
& \left|H_{j}\left(x_{1}, y_{1}, r, p\right)-H_{j}\left(x_{2}, y_{2}, r, p\right)\right| \leq \omega\left((1+|p|)\left(\left|x_{1}-x_{2}\right|+\left|y_{1}-y_{2}\right|\right)\right), \\
& \text { for every } x_{1}, x_{2}, y_{1}, y_{2}, p \in \mathbb{R}^{N} \text { and } r \in \mathbb{R}^{M} .
\end{aligned}
$$


Unless otherwise specified all the periodic functions we consider have period $\mathbb{T}^{N}=[0,1]^{N}$. We also assume the following monotonicity condition

$$
\begin{aligned}
& \text { If } r, s \in \mathbb{R}^{M} \text { and } r_{j}-s_{j}=\max _{k=1, \ldots, M}\left\{r_{k}-s_{k}\right\} \geq 0 \text {, then } \\
& \text { for all } x, y, p \in \mathbb{R}^{N}, \quad H_{j}(x, y, r, p)-H_{j}(x, y, s, p) \geq 0 .
\end{aligned}
$$

Concerning the initial datum we assume

$$
u_{0, j} \text { is bounded uniformly continuous in } \mathbb{R}^{N} \text { for } j=1, \ldots, M \text {. }
$$

Example 2.1. (1) Consider

$$
H_{j}(x, y, r, p)=a_{j}(x, y)|p|+F_{j}(r)
$$

where $a_{j} \in C\left(\mathbb{R}^{N} \times \mathbb{R}^{N}\right)$ and $F_{j} \in C\left(\mathbb{R}^{M}\right)$. If $a_{j}$ is $\mathbb{Z}^{N}$-periodic in $y$, then (2.2) (i) holds. If there exists $\delta>0$ such that $a_{j} \geq \delta$ then (2.2) (ii) is satisfied. If $a_{j}$ is bounded with respect to $x$ then (2.2) (iii) holds (note that $a_{j}$ is bounded with respect to $y$ since it is periodic). Finally, we have (2.2) (iv) if, for instance, $a_{j}$ is Lipschitz continuous with respect to $(x, y)$. The assumption $(2.3)$ is satisfied if $F_{j}$ is increasing in $r_{j}$, decreasing in $r_{k}$ for $k \neq j$.

(2) A weakly coupled system is a system of the type

$$
\frac{\partial u_{i}^{\varepsilon}}{\partial t}+H_{i}\left(x, \frac{x}{\varepsilon}, D u_{i}^{\varepsilon}\right)+\sum_{j=1}^{M} c_{j i}\left(x, \frac{x}{\varepsilon}\right) u_{j}=0, \quad i=1, \ldots, M .
$$

Some assumptions on $c_{i j}$ to ensure (2.3) are given in Section 6. Weakly coupled systems arise in optimal control theory of random evolution processes (see [10]). Moreover they are associated to large deviation theory for small random perturbations of a random evolution process (see $[6,9]$ ). We will study some specific case of weakly coupled systems in Section 6.

For a function $u: E \rightarrow \mathbb{R}^{M}$, we say that $u=\left(u_{1}, \ldots, u_{M}\right)$ is upper-semicontinuous (u.s.c. in short), respectively lower-semicontinuous (l.s.c. in short), in $E$ if all the components $u_{i}, i=1, \ldots, M$, are u.s.c., respectively l.s.c., in $E$. We define in the same way bounded uniformly continuous $(B U C)$ and Lipschitz continuous functions $u: E \rightarrow \mathbb{R}^{M}$. If $u=\left(u_{1}, \ldots, u_{M}\right), v=\left(v_{1}, \ldots, v_{M}\right)$, are two functions defined in a set $E$ we write $u \leq v$ in $E$ if $u_{i} \leq v_{i}$ in $E$ for all $i \in\{1, \ldots, M\}$.

We recall the definition of viscosity solution for the system (2.1).

\section{Definition 2.1.}

(i) An u.s.c. function $u: \mathbb{R}^{N} \times(0, T) \rightarrow \mathbb{R}^{M}$ is said a viscosity subsolution of $(2.1)$ if $u_{i}(\cdot, 0) \leq u_{0, i}$ in $\mathbb{R}^{N}$ for all $i \in\{1, \ldots, M\}$ and if whenever $\phi \in C^{1}, i \in\{1, \ldots, M\}$ and $u_{i}-\phi$ attains a local maximum at $(x, t)$ with $t>0$, then

$$
\frac{\partial \phi}{\partial t}(x, t)+H_{i}\left(x, \frac{x}{\varepsilon}, u(x, t), D \phi(x, t)\right) \leq 0 .
$$

(ii) A l.s.c. function $v: \mathbb{R}^{N} \times(0, T) \rightarrow \mathbb{R}^{M}$ is said a viscosity supersolution of $(2.1)$ if $v_{i}(\cdot, 0) \geq u_{0, i}$ in $\mathbb{R}^{N}$ for all $i \in\{1, \ldots, M\}$ and if whenever $\phi \in C^{1}, i \in\{1, \ldots, M\}$ and $v_{i}-\phi$ attains a local minimum at $(x, t)$ with $t>0$, then

$$
\frac{\partial \phi}{\partial t}(x, t)+H_{i}\left(x, \frac{x}{\varepsilon}, v(x, t), D \phi(x, t)\right) \geq 0 .
$$

(iii) A continuous function $u$ is said a viscosity solution of (2.1) if it is both a viscosity sub- and supersolution of (2.1). 


\section{The evolutive Problem For $\varepsilon>0$}

In this section we study the system (2.1) for $\varepsilon>0$ fixed. We first prove a comparison theorem which applies to prove existence and uniqueness for (2.1). Without loss of generality, we can skip the $y$-dependence in the Hamiltonians below and we prove a slightly more general result for $H_{j}=H_{j}(x, t, u, p)$ which depends also on $t$ (and is continuous in $\mathbb{R}^{N} \times[0, T] \times \mathbb{R}^{M} \times \mathbb{R}^{N}$ ).

Proposition 3.1. Let $u$ be a bounded u.s.c. subsolution and $v$ be a bounded l.s.c. supersolution of

$$
\begin{cases}\frac{\partial u_{i}}{\partial t}+H_{i}\left(x, t, u, D u_{i}\right)=0 & (x, t) \in \mathbb{R}^{N} \times(0, T) \\ u_{i}(x, 0)=u_{0, i}(x) & x \in \mathbb{R}^{N}, \quad i=1, \ldots, M\end{cases}
$$

where $H_{i}$ satisfies (2.2)-(2.3) and $u_{0}$ satisfies (2.4). Then $u \leq v$ in $\mathbb{R}^{N} \times[0, T]$ and there exists a unique continuous viscosity solution $u$ of (3.1).

Proof. We first prove the comparison principle. Define

$$
\Psi(x, y, t, s, j)=u_{j}(x, t)-v_{j}(y, s)-\frac{|x-y|^{2}}{2 \alpha}-\frac{|t-s|^{2}}{2 \mu}-\beta\left(|x|^{2}+|y|^{2}\right)-\eta t,
$$

where $\alpha, \beta, \mu, \eta$ are positive constants. Since $u, v$ are bounded, $\max _{j} \sup _{\left(\mathbb{R}^{N}\right)^{2} \times[0, T]} \Psi$ is finite and achieved at some $(\bar{x}, \bar{y}, \bar{t}, \bar{s}, \bar{j})$.

For all $j$ and $(x, t) \in \mathbb{R}^{N}$, we have

$$
u_{j}(x, t)-v_{j}(x, t)-2 \beta|x|^{2}-\eta t=\Psi(x, x, t, t, j) \leq \Psi(\bar{x}, \bar{y}, \bar{t}, \bar{s}, \bar{j}) \leq u_{\bar{j}}(\bar{x}, \bar{t})-v_{\bar{j}}(\bar{y}, \bar{s}) .
$$

If $u_{\bar{j}}(\bar{x}, \bar{t})-v_{\bar{j}}(\bar{y}, \bar{s}) \leq 0$ for all $\beta, \eta>0$, then the comparison holds. Therefore, we suppose that

$$
u_{\bar{j}}(\bar{x}, \bar{t})-v_{\bar{j}}(\bar{y}, \bar{s}) \geq 0
$$

for $\beta, \eta$ sufficiently small.

The following inequality

$$
u_{1}(0,0)-v_{1}(0,0)=\Psi(0,0,0,0,1) \leq \Psi(\bar{x}, \bar{y}, \bar{t}, \bar{s}, \bar{j})
$$

and the boundedness of $u, v$ leads to the classical estimates (see [3], Lem. 4.3)

$$
\begin{aligned}
& \beta\left(|\bar{x}|^{2}+|\bar{y}|^{2}\right), \frac{|\bar{t}-\bar{s}|^{2}}{2 \mu}, \frac{|\bar{x}-\bar{y}|^{2}}{2 \alpha} \leq 2\left(|u|_{\infty}+|v|_{\infty}\right), \\
& \lim _{\mu \rightarrow 0} \frac{|\bar{t}-\bar{s}|^{2}}{2 \mu}=0, \quad \lim _{\beta \rightarrow 0} \beta(|\bar{x}|+|\bar{y}|)=0 \quad \text { and } \quad \lim _{\alpha \rightarrow 0} \limsup _{\mu, \beta \rightarrow 0} \frac{|\bar{x}-\bar{y}|^{2}}{2 \alpha}=0
\end{aligned}
$$

we will need later.

Assume for a while that it is possible to extract some subsequences $\alpha, \beta, \mu \rightarrow 0$ such that

$$
\bar{t}>0 \text { and } \bar{s}>0 \text {. }
$$

It follows that we can write the viscosity inequalities for the subsolution $u$ and the supersolution $v$. Setting $\bar{p}=(\bar{x}-\bar{y}) / \alpha$, we have

$$
\frac{(\bar{t}-\bar{s})}{\alpha}+H_{\bar{j}}(\bar{x}, \bar{t}, u(\bar{x}, \bar{t}), \bar{p}+2 \beta \bar{x}) \leq 0
$$


and

Subtracting (3.7) from (3.6), we obtain

$$
\frac{(\bar{t}-\bar{s})}{\alpha}+H_{\bar{j}}(\bar{y}, \bar{s}, v(\bar{y}, \bar{s}), \bar{p}-2 \beta \bar{y}) \geq 0 .
$$

$$
\eta \leq H_{\bar{j}}(\bar{y}, \bar{s}, v(\bar{y}, \bar{s}), \bar{p}-2 \beta \bar{y})-H_{\bar{j}}(\bar{x}, \bar{t}, u(\bar{x}, \bar{t}), \bar{p}+2 \beta \bar{x})=\mathcal{T}_{1}+\mathcal{T}_{2}+\mathcal{T}_{3}+\mathcal{T}_{4},
$$

where

$$
\begin{aligned}
& \mathcal{T}_{1}=H_{\bar{j}}(\bar{y}, \bar{s}, v(\bar{y}, \bar{s}), \bar{p}-2 \beta \bar{y})-H_{\bar{j}}(\bar{x}, \bar{s}, v(\bar{y}, \bar{s}), \bar{p}-2 \beta \bar{y}), \\
& \mathcal{T}_{2}=H_{\bar{j}}(\bar{x}, \bar{s}, v(\bar{y}, \bar{s}), \bar{p}-2 \beta \bar{y})-H_{\bar{j}}(\bar{x}, \bar{t}, v(\bar{y}, \bar{s}), \bar{p}-2 \beta \bar{y}), \\
& \mathcal{T}_{3}=H_{\bar{j}}(\bar{x}, \bar{t}, v(\bar{y}, \bar{s}), \bar{p}-2 \beta \bar{y})-H_{\bar{j}}(\bar{x}, \bar{t}, v(\bar{y}, \bar{s}), \bar{p}+2 \beta \bar{x}), \\
& \mathcal{T}_{4}=H_{\bar{j}}(\bar{x}, \bar{t}, v(\bar{y}, \bar{s}), \bar{p}+2 \beta \bar{x})-H_{\bar{j}}(\bar{x}, \bar{t}, u(\bar{x}, \bar{t}), \bar{p}+2 \beta \bar{x}) .
\end{aligned}
$$

From (3.3), choosing $0<\alpha, \beta<1$ and setting $M=\sqrt{2\left(|u|_{\infty}+|v|_{\infty}\right)}$ we have

$$
\beta|\bar{x}|, \beta|\bar{y}| \leq M \sqrt{\beta} \leq M \quad \text { and } \quad|\bar{p}| \leq \frac{M}{\sqrt{\alpha}}
$$

From (2.2) (iv), we have

$$
\begin{aligned}
\mathcal{T}_{1} & \leq \omega((1+|\bar{p}|+2 \beta|\bar{y}|)|\bar{y}-\bar{x}|) \\
& \leq \omega\left((1+2 M) M \sqrt{\alpha}+\frac{|\bar{y}-\bar{x}|^{2}}{\alpha}\right) .
\end{aligned}
$$

If $\alpha, \beta$ are fixed, $\bar{x}, v(\bar{y}, \bar{s}), p-2 \beta \bar{y}$ are bounded independently of $\mu$ by (3.3). It follows that there exists a modulus of continuity $\omega_{\alpha, \beta,|v|_{\infty}, T}$ such that

$$
\mathcal{T}_{2} \leq \omega_{\alpha, \beta,|v|_{\infty}, T}(|\bar{s}-\bar{t}|) .
$$

By (3.9),

$$
|\bar{p}-2 \beta \bar{y}|,|\bar{p}+2 \beta \bar{x}| \leq \frac{M}{\sqrt{\alpha}}+2 M
$$

and therefore, by (2.2) (iii), there exists a modulus of continuity $\omega_{\alpha,|v|_{\infty}, T}$ such that

$$
\mathcal{T}_{3} \leq \omega_{\alpha,|v|_{\infty}, T}(\beta(|\bar{x}|+|\bar{y}|)) \leq \omega_{\alpha,|v|_{\infty}, T}(2 M \beta) .
$$

The non classical term here is $\mathcal{T}_{4}$ for which we have to use (2.3) to deal with: since $0 \leq u_{\bar{j}}(\bar{x}, \bar{t})-v_{\bar{j}}(\bar{y}, \bar{s})=$ $\max _{1 \leq i \leq M}\left\{u_{i}(\bar{x}, \bar{t})-v_{i}(\bar{y}, \bar{s})\right\}$ by definition of $\bar{j}$ and (3.2), we obtain

$$
\mathcal{T}_{4} \leq 0 .
$$

Finally, (3.8) reads

$$
\eta \leq \omega\left((1+2 M) M \sqrt{\alpha}+\frac{|\bar{y}-\bar{x}|^{2}}{\alpha}\right)+\omega_{\alpha, \beta,|v|_{\infty}, T}(|\bar{s}-\bar{t}|)+\omega_{\alpha,|v|_{\infty}, T}(\beta(|\bar{x}|+|\bar{y}|)) .
$$

By (3.4), we can take $\alpha$ small enough to have $\omega(\ldots) \leq \eta / 3$. Then we choose successively $\beta$ and $\mu$ small enough to obtain a contradiction in (3.11). 
Therefore, choosing $\mu \ll \beta \ll \alpha \ll \eta$ small enough, (3.5) does not hold and, for all extractions, one has for instance $\bar{t}=0$. It follows that, for all $j$ and $x, y \in \mathbb{R}^{N}, t \in[0, T]$, we have

$$
u_{j}(x, t)-v_{j}(y, t) \leq \eta t+\frac{|x-y|^{2}}{2 \alpha}+\beta\left(|x|^{2}+|y|^{2}\right)+\left(u_{\bar{j}}(\bar{x}, 0)-v_{\bar{j}}(\bar{y}, \bar{s})\right)^{+}-\frac{|\bar{x}-\bar{y}|^{2}}{2 \alpha} .
$$

Sending $\mu \rightarrow 0$ and then $\beta \rightarrow 0$, we obtain, using (3.4),

$$
u_{j}(x, t)-v_{j}(y, t) \leq \eta t+\frac{|x-y|^{2}}{2 \alpha}+\left(u_{\bar{j}}(\bar{x}, 0)-v_{\bar{j}}(\bar{y}, 0)\right)^{+}-\frac{|\bar{x}-\bar{y}|^{2}}{2 \alpha} .
$$

But $u_{\bar{j}}(\bar{x}, 0)-v_{\bar{j}}(\bar{y}, 0) \leq u_{0, \bar{j}}(\bar{x})-u_{0, \bar{j}}(\bar{y})$ and by uniform continuity of the $u_{0, j}$ 's, $j=1, \ldots, M$, for all $\rho>0$, there exists $C_{j, \rho}>0$ such that

$$
u_{0, j}(x)-u_{0, j}(y) \leq \rho+C_{j, \rho}|x-y|
$$

and therefore

$$
u_{0, j}(x)-u_{0, j}(y)-\frac{|x-y|^{2}}{2 \alpha} \leq \rho+C_{j, \rho}|x-y|-\frac{|x-y|^{2}}{2 \alpha} \leq \rho+\frac{1}{2} \alpha C_{j, \rho}^{2} .
$$

We fix $\rho>0$ and set $C_{\rho}=\max _{1 \leq i \leq M} C_{i, \rho} / \sqrt{2}$. Then (3.12) becomes

$$
u_{j}(x, t)-v_{j}(y, t) \leq \rho+\eta t+\alpha C_{\rho}^{2}+\frac{|x-y|^{2}}{2 \alpha}
$$

Using (3.4) and sending successively $\alpha \rightarrow 0, \eta \rightarrow 0, \rho \rightarrow 0$, we conclude that the comparison holds.

By classical Perron's method (see [14]), comparison implies the existence of a continuous viscosity solution $u$ to (3.1). Applying the comparison principle again, we obtain the uniqueness of the solution.

Proposition 3.2. Under the assumptions of Proposition 3.1, let $u$ be the unique bounded continuous viscosity solution of (3.1). Then $u \in B U C\left(\mathbb{R}^{N} \times[0, T]\right)$.

Proof. We first prove that $u$ is bounded. Let

$$
u^{ \pm}(x, t)=\left( \pm\left|u_{0}\right|_{\infty} \pm C t, \ldots, \pm\left|u_{0}\right|_{\infty} \pm C t\right),
$$

where $C=C\left(H,\left|u_{0}\right|_{\infty}, T\right)$ is defined by

$$
C:=\sup \left\{\left|H_{j}(x, t, r, 0)\right|: x \in \mathbb{R}^{N}, t \in[0, T],|r| \leq\left|u_{0}\right|_{\infty}, 1 \leq j \leq M\right\} .
$$

It suffices to prove that $u^{+}$is a supersolution and $u^{-}$a subsolution of (3.1). Then, by the comparison principle of Proposition 3.1, we get

$$
u^{-} \leq u \leq u^{+} \quad \mathbb{R}^{N} \times[0, T]
$$

and we obtain the global $L^{\infty}$ bound

$$
|u| \leq\left|u_{0}\right|_{\infty}+C\left(H,\left|u_{0}\right|_{\infty}, T\right) T
$$

We only prove that $u^{+}$is a supersolution, the proof for $u^{-}$being similar. At first, $u^{+}$satisfies clearly the initial condition. Since $u^{+}$is smooth, for all $j$ and $(x, t) \in \mathbb{R}^{N} \times(0, T)$,

$$
\frac{\partial u_{j}^{+}}{\partial t}+H_{j}\left(x, t, u^{+}(x, t), D u_{j}^{+}(x, t)\right)=C+H_{j}\left(x, t, u^{+}(x, t), 0\right) .
$$


But

$$
\max _{1 \leq k \leq M}\left\{u_{k}^{+}(x, t)-\left|u_{0}\right|_{\infty}\right\}=C t \geq 0
$$

is achieved for every index $1 \leq k \leq M$. Therefore, from (2.3), for all $j$,

$$
H_{j}\left(x, t, u^{+}(x, t), 0\right) \geq H_{j}\left(x, t,\left(\left|u_{0}\right|_{\infty}, \ldots,\left|u_{0}\right|_{\infty}\right), 0\right) \geq-C
$$

which proves the result.

We prove the uniform continuity of $u$ in the space variable uniformly in time. Repeating the proof of the comparison principle with $v=u$, from (3.15), we obtain for all $\rho, \eta>0$, there exists $C_{\rho}$ such that, for all $j$, $x, y \in \mathbb{R}^{N}$, and $t \in[0, T]$,

$$
u_{j}(x, t)-u_{j}(y, t) \leq \rho+\eta t+\inf _{\alpha>0}\left\{\alpha C_{\rho}^{2}+\frac{|x-y|^{2}}{2 \alpha}\right\} \leq 2 \rho+\sqrt{2} C_{\rho}|x-y|
$$

if we take $\eta$ such that $\eta T \leq \rho$. This proves that there exists a modulus of continuity $\omega_{\text {sp }}$ in space for $u$ which is independent of $t \in[0, T]$ :

$$
u_{j}(x, t)-u_{j}(y, t) \leq \omega_{\mathrm{sp}}(|x-y|) \quad x, y \in \mathbb{R}^{N}, t \in[0, T]
$$

We continue by deducing a modulus of continuity in time (uniformly in space). This result is classical in parabolic PDEs. Here we adapt the proof of [5], Lemma 9.1. We want to prove that, for all $\rho>0$, there exist positive constants $C_{\rho}$ and $K_{\rho}$ such that, for all $j, x, x_{0} \in \mathbb{R}^{N}$ with $\left|x-x_{0}\right| \leq 1$ and $0 \leq t_{0} \leq t \leq T$,

$$
u_{j}(x, t)-u_{j}\left(x_{0}, t_{0}\right) \leq \rho+C_{\rho}\left|x-x_{0}\right|^{2}+K_{\rho}\left(t-t_{0}\right)
$$

and

$$
-\rho-C_{\rho}\left|x-x_{0}\right|^{2}-K_{\rho}\left(t-t_{0}\right) \leq u_{j}(x, t)-u_{j}\left(x_{0}, t_{0}\right)
$$

We will prove only the first inequality, the proof of the second one being analogous. Since $x \in \bar{B}\left(x_{0}, 1\right)$, taking

$$
C_{\rho} \geq 2|u|_{\infty}
$$

we are sure that $(3.18)$ holds on $\partial B\left(x_{0}, 1\right) \times\left[t_{0}, T\right]$ for every $\rho, K_{\rho}>0$. It is worth noticing that $C_{\rho}$ depends only on $|u|_{\infty}$. Next we would like to ensure that (3.18) holds in $\bar{B}\left(x_{0}, 1\right) \times\left\{t_{0}\right\}$. To this end, we argue by contradiction assuming there exists $\rho>0$ such that, for every $C_{\rho}>0$, there exists $j$ and $y_{C_{\rho}} \in \bar{B}\left(x_{0}, 1\right)$ with

$$
u_{j}\left(y_{C_{\rho}}, t_{0}\right)-u_{j}\left(x_{0}, t_{0}\right)>\rho+C_{\rho}\left|y_{C_{\rho}}-x_{0}\right|^{2} .
$$

It follows

$$
\left|y_{C_{\rho}}-x_{0}\right| \leq \sqrt{\frac{2|u|_{\infty}}{C_{\rho}}} \rightarrow 0 \text { as } C_{\rho} \rightarrow+\infty
$$

From (3.20), we get

$$
\omega_{\mathrm{sp}}\left(\left|y_{C_{\rho}}-x_{0}\right|\right) \geq u_{j}\left(y_{C_{\rho}}, t_{0}\right)-u_{j}\left(x_{0}, t_{0}\right)>\rho+C_{\rho}\left|y_{C_{\rho}}-x_{0}\right|^{2} \geq \rho
$$


which leads to a contradiction for $C_{\rho}$ large enough. Note that the choice of $C_{\rho}$ to obtain the contradiction depends only on $\rho,|u|_{\infty}$ and $\omega_{\text {sp }}$. Finally we proved that, up to choose $C_{\rho}=C_{\rho}\left(\rho,|u|_{\infty}, \omega_{\mathrm{sp}}\right)$ big enough, (3.18) holds on $\left(\partial B\left(x_{0}, 1\right) \times\left[t_{0}, T\right]\right) \cup\left(\bar{B}\left(x_{0}, 1\right) \times\left\{t_{0}\right\}\right)$.

For all $1 \leq j \leq M$, we set

$$
\chi_{j}(y, t):=u_{j}\left(x_{0}, t_{0}\right)+\rho+C_{\rho}\left|y-x_{0}\right|^{2}+K_{\rho}\left(t-t_{0}\right) \quad(y, t) \in \mathbb{R}^{N} \times[0, T]
$$

and $\chi=\left(\chi_{1}, \ldots, \chi_{M}\right)$. Note that $\chi$ is a smooth function. We claim that we can choose the constant $K_{\rho}$ big enough in order that $\chi$ is a strict supersolution of $(3.1)$ in $B\left(x_{0}, 1\right) \times\left(t_{0}, T\right)$. Indeed, for all $j$, and $(y, t) \in$ $B\left(x_{0}, 1\right) \times\left(t_{0}, T\right)$,

$$
\frac{\partial \chi_{j}}{\partial t}+H_{j}\left(y, t, \chi(y, t), D \chi_{j}(y, t)\right)=K_{\rho}+H_{j}\left(y, t, \chi(y, t), 2 C_{\rho}\left(y-x_{0}\right)\right)
$$

But

$$
\max _{1 \leq k \leq M}\left\{\chi_{k}(y, t)-u_{k}\left(x_{0}, t_{0}\right)\right\}=\rho+C_{\rho}\left|y-x_{0}\right|^{2}+K_{\rho}\left(t-t_{0}\right) \geq 0
$$

is achieved for every index $1 \leq k \leq M$. Therefore, from (2.3), for all $j$,

$$
H_{j}\left(y, t, \chi(y, t), 2 C_{\rho}\left(y-x_{0}\right)\right) \geq H_{j}\left(y, t, u\left(x_{0}, t_{0}\right), 2 C_{\rho}\left(y-x_{0}\right)\right) .
$$

By (2.2) (iii),

$$
M_{C_{\rho},|u|_{\infty}}:=\inf \left\{H_{j}(y, t, r, p): y \in \mathbb{R}^{N}, t \in[0, T],|r| \leq|u|_{\infty},|p| \leq 2 C_{\rho}, 1 \leq j \leq M\right\}
$$

is finite. Taking

$$
K_{\rho}>-M_{C_{\rho},|u|_{\infty}},
$$

from (3.21) and (3.22), we obtain, for all $j$,

$$
\frac{\partial \chi_{j}}{\partial t}+H_{j}\left(y, t, \chi(y, t), D \chi_{j}(y, t)\right)>0 \quad(y, t) \in B\left(x_{0}, 1\right) \times\left(t_{0}, T\right)
$$

which proves the claim.

From the very definition of viscosity solution, it follows that, for all $j, \max _{\bar{B}\left(x_{0}, 1\right) \times\left(t_{0}, T\right)}\left\{u_{j}-\chi_{j}\right\}$ is necessarily achieved on the parabolic boundary of $B\left(x_{0}, 1\right) \times\left(t_{0}, T\right)$ and therefore (3.18) holds in $\bar{B}\left(x_{0}, R\right) \times\left[t_{0}, T\right]$.

From (3.18) and (3.19), we obtain that, for all $\rho>0,1 \leq j \leq M, x \in \mathbb{R}^{N}$, and $t, s \in[0, T]$,

$$
\left|u_{j}(x, t)-u_{j}(x, s)\right| \leq \rho+K_{\rho}|t-s|
$$

and $K_{\rho}$ is independent of $x$. This proves the existence of a modulus of continuity $\omega_{\mathrm{tm}}$ in time which is independent of $x \in \mathbb{R}^{N}$.

In the following proposition, we prove some a-priori bounds, independent of $\varepsilon$, which are used in the homogenization theorem.

Proposition 3.3. Assume (2.2), (2.3) and (2.4). For any $\varepsilon>0$ there exists a unique solution $u^{\varepsilon} \in B U C\left(\mathbb{R}^{N} \times\right.$ $[0, T])$ of (2.1). Moreover

(i) If $u_{0}$ is bounded Lipschitz continuous, then $u^{\varepsilon} \in W^{1, \infty}\left(\mathbb{R}^{N} \times[0, T]\right)$ and $\left|D u^{\varepsilon}\right|_{\infty}$ can be bounded independently of $\varepsilon$. 
(ii) If $u_{0}$ is $B U C$, then

$$
\begin{aligned}
& \left|u^{\varepsilon}\right|_{\infty} \leq L\left(H_{i},\left|u_{0}\right|_{\infty}, T\right) \\
& \left|u^{\varepsilon}(x, t)-u^{\varepsilon}(y, s)\right| \leq \omega_{\mathrm{sp}}(|x-y|)+\omega_{\mathrm{tm}}(|t-s|) \quad x, y \in \mathbb{R}^{N}, t, s \in[0, T]
\end{aligned}
$$

and $L\left(H_{i},\left|u_{0}\right|_{\infty}, T\right), \omega_{\mathrm{sp}}, \omega_{\mathrm{tm}}$ are independent of $\varepsilon$.

Proof. For fixed $\varepsilon>0$, the existence and uniqueness of the solution $u^{\varepsilon} \in B U C\left(\mathbb{R}^{N} \times[0, T]\right)$ to (2.1) follows immediately by Propositions 3.1 and 3.2. Note that the $L^{\infty}$ bound for $u^{\varepsilon}$ does not depend on $\varepsilon$. Indeed, replacing $C$ in (3.16) by

$$
C:=\sup \left\{\left|H_{j}\left(x, \frac{y}{\varepsilon}, r, 0\right)\right|: x \in \mathbb{R}^{N}, y \in \mathbb{R}^{N},|r| \leq\left|u_{0}\right|_{\infty}, 1 \leq j \leq M\right\}
$$

which is finite and independent of $\varepsilon$ by periodicity of $H_{j}$ in $y$, we obtain (3.17) and therefore (3.23).

We now prove (3.24). Let $u^{\varepsilon}$ be a subsolution and $v^{\varepsilon}$ be a supersolution of (2.1) which are BUC (the modulus of continuity of the solution may a priori depend of $\varepsilon$ ). Arguing as in Proposition 3.1, from (3.12) we have, for all $j$ and $(x, t) \in \mathbb{R}^{N} \times[0, T]$,

$$
\begin{aligned}
u_{j}^{\varepsilon}(x, t)-v_{j}^{\varepsilon}(x, t) & \leq \eta t+\left(u \frac{\varepsilon}{\bar{j}}(\bar{x}, 0)-v \bar{\varepsilon}(\bar{y}, 0)\right)^{+}-\frac{|\bar{x}-\bar{y}|^{2}}{2 \alpha} \\
& \leq \eta T+\max _{1 \leq j \leq M} \sup _{\mathbb{R}^{N}}\left(u_{j}^{\varepsilon}(\cdot, 0)-v_{j}^{\varepsilon}(\cdot, 0)\right)^{+}+\left(v \frac{\varepsilon}{\bar{j}}(\bar{x}, 0)-v \frac{\varepsilon}{\bar{j}}(\bar{y}, 0)\right)^{+}-\frac{|\bar{x}-\bar{y}|^{2}}{2 \alpha}
\end{aligned}
$$

and

$$
\limsup _{\alpha \rightarrow 0}\left(v \frac{\varepsilon}{\bar{j}}(\bar{x}, 0)-v \frac{\varepsilon}{j}(\bar{y}, 0)\right)^{+}-\frac{|\bar{x}-\bar{y}|^{2}}{2 \alpha} \leq 0
$$

since $v$ is uniformly continuous (see (3.13) and (3.14)). Letting $\alpha \rightarrow 0$ and then $\eta \rightarrow 0$, we obtain

$$
\max _{1 \leq j \leq M} \sup _{\mathbb{R}^{N} \times[0, T]} u_{j}^{\varepsilon}-v_{j}^{\varepsilon} \leq \max _{1 \leq j \leq M} \sup _{\mathbb{R}^{N}}\left(u_{j}^{\varepsilon}(\cdot, 0)-v_{j}^{\varepsilon}(\cdot, 0)\right)^{+} .
$$

We have to prove that the modulus of continuity of $u^{\varepsilon}$ do not depend on $\varepsilon$. We proceed by approximation showing first the result for $u_{0}$ Lipschitz continuous. Replacing $C$ in (3.16) by

$$
C:=\sup \left\{\left|H_{j}\left(x, \frac{y}{\varepsilon}, r, p\right)\right|: x \in \mathbb{R}^{N}, y \in \mathbb{R}^{N},|r| \leq\left|u_{0}\right|_{\infty},|p| \leq\left|D u_{0}\right|_{\infty}, 1 \leq j \leq M\right\},
$$

we prove as at the beginning of the proof of Proposition 3.2 that, if $v^{ \pm}(x, t)=\left(u_{0,1}(x) \pm C t, \ldots, u_{0, M}(x) \pm C t\right)$, then $v^{+}$is a supersolution and $v^{-}$is a subsolution of (2.1). By Proposition 3.1, it follows

$$
v^{-} \leq u \leq v^{+} \text {in } \mathbb{R}^{N} \times[0, T] .
$$

Let $0 \leq h \leq T$ and note that, since the $H_{i}$ 's are independent of $t, u^{\varepsilon}(\cdot, \cdot+h)$ is still a solution of (2.1) with initial data $u^{\varepsilon}(\cdot, h)$. By (3.25) and (3.26), we get for all $j,(x, t) \in \mathbb{R}^{N} \times[0, T]$

$$
u_{j}^{\varepsilon}(x, t+h)-u_{j}^{\varepsilon}(x, t) \leq \max _{1 \leq j \leq M} \sup _{\mathbb{R}^{N}}\left(u_{j}^{\varepsilon}(\cdot, h)-u_{0, j}\right)^{+} \leq C h
$$

and therefore $u_{j}^{\varepsilon}$ is Lipschitz with respect to $t$ for every $x$ with

$$
\left|\frac{\partial u_{j}^{\varepsilon}}{\partial t}\right|_{\infty} \leq C
$$


From (2.1), we obtain, in the viscosity sense

$$
-C \leq H_{j}\left(x, \frac{x}{\varepsilon}, u^{\varepsilon}, D u_{j}^{\varepsilon}\right) \leq C \quad(x, t) \in \mathbb{R}^{N} \times[0, T] .
$$

By the coercivity of $H_{i}$ (uniformly with respect to the other variables, see (2.2) (ii)), there exists $L_{j}>0$ such that, for all $p \in \mathbb{R}^{N}$,

$$
|p| \geq L_{j} \Longrightarrow \text { for all } \varepsilon>0, x \in \mathbb{R}^{N}, r \in \mathbb{R}^{M}, H_{j}\left(x, \frac{x}{\varepsilon}, r, p\right)>C .
$$

It follows that $u_{j}^{\varepsilon}$ is Lipschitz continuous in $x$ for every $t$ with $\left|D u_{j}^{\varepsilon}\right|_{\infty} \leq L_{j}$ (with $L_{j}$ independent of $\varepsilon$ ).

Now if $u_{0} \in B U C\left(\mathbb{R}^{N}\right)$, then it is possible to approach it by Lipschitz continuous functions: for all $\gamma>0$, there exists $u_{0}^{\gamma}$ such that $\left|u_{0}-u_{0}^{\gamma}\right|_{\infty} \leq \gamma$. Let $u^{\varepsilon}$ (respectively $u^{\varepsilon, \gamma}$ ) be the unique BUC (respectively Lipschitz continuous with constant $C_{\gamma}$ ) solution of (2.1) with initial data $u_{0}$ (respectively $u_{0}^{\gamma}$ ). Note that $C_{\gamma}$ is independent of $\varepsilon$. By (3.25), we obtain

$$
\left|u^{\varepsilon}-u^{\varepsilon, \gamma}\right|_{\infty} \leq\left|u_{0}-u_{0}^{\gamma}\right|_{\infty} \leq \gamma .
$$

It follows that, for all $1 \leq j \leq M, x, y \in \mathbb{R}^{N}, t, s \in[0, T]$,

$$
\left|u_{j}^{\varepsilon}(x, t)-u_{j}^{\varepsilon}(y, s)\right| \leq\left|u_{j}^{\varepsilon, \gamma}(x, t)-u_{j}^{\varepsilon, \gamma}(y, s)\right|+2 \gamma \leq C_{\gamma}(|x-y|+|t-s|)+2 \gamma .
$$

Since (3.27) holds for all $\gamma>0$ and $C_{\gamma}$ is independent of $\varepsilon$, we conclude that $u^{\varepsilon}$ is $B U C$ with a modulus independent of $\varepsilon$.

\section{The CELL PROBLEM}

In this section we prove the existence of the effective Hamiltonians, the Hamiltonians for the limit system (1.2). Since at this level we work for a fixed index $i$, i.e. there is no coupling, we can follow the classical argument based on the ergodic approximation of the cell problem. The only point is to prove that effective Hamiltonians we are going to define still verify some regularity and monotonicity properties so that the homogenized problem verifies a comparison principle.

The cell problem. For any $i=1, \ldots, M$, given $(x, r, p) \in \mathbb{R}^{N} \times \mathbb{R}^{M} \times \mathbb{R}^{N}$, find $\lambda_{i}=\lambda_{i}(x, r, p)$ such that the equation

admits a viscosity solution $v_{i}=v_{i, x, r, p}$

$$
H_{i}(x, y, r, p+D v(y))=\lambda_{i} \quad y \in \mathbb{T}^{N}
$$

Proposition 4.1. Assume (2.2). For any $i=1, \ldots, M$, there exists a unique $\lambda_{i}=\lambda_{i}(x, r, p) \in \mathbb{R}$ such that the cell problem (4.1) admits a periodic solution $v_{i}(y)=v_{i}(y ; x, r, p)$ which is Lipschitz continuous. More precisely, for all $R>0$, there exists $L_{R}>0$ such that

$$
\sup \left\{\left|D_{y} v_{i}(y ; x, r, p)\right|_{\infty}: x \in \mathbb{R}^{N},|r|+|p| \leq R\right\} \leq L_{R} .
$$

Proof. We only give a sketch of the proof, see for instance [8] for details. Fix $i \in\{1, \ldots, M\}, R>0$ and $(x, r, p) \in \mathbb{R}^{N} \times \mathbb{R}^{M} \times \mathbb{R}^{N}$ such that $|r|+|p| \leq R$. Consider the ergodic approximation of the cell problem

$$
\alpha w_{i}^{\alpha}(y)+H_{i}\left(x, y, r, p+D w_{i}^{\alpha}(y)\right)=0 \quad y \in \mathbb{T}^{N} .
$$

By (2.2), (4.2) satisfies a comparison principle for any $\alpha>0$ and therefore it admits a unique continuous viscosity solution $w_{i}^{\alpha}$ which is periodic. By (2.2) (iii),

$$
C_{R}:=\sup \left\{\left|H_{j}(x, y, r, p)\right|: x, y \in \mathbb{R}^{N},|r|+|p| \leq R, j=1, \ldots, M\right\}<+\infty
$$


and $-C_{R} / \alpha$ is a subsolution and $C_{R} / \alpha$ is a supersolution of (4.2). It follows

$$
-C_{R} \leq \alpha w_{i}^{\alpha} \leq C_{R} .
$$

By the coercitivity of the Hamiltonian $H_{i}$, there exists $L_{R}=L\left(R, H_{i}\right)$ such that

$$
\left|p+D w_{i}^{\alpha}\right|>L_{R} \quad \Longrightarrow \quad H_{i}\left(x, y, r, p+D w_{i}^{\alpha}\right)>C_{R} .
$$

We then get the global gradient bounds for $w_{i}^{\alpha}$ independent of $\alpha$ :

$$
\left|D w_{i}^{\alpha}\right|_{\infty} \leq L_{R}
$$

It follows that, for a fixed $y_{0} \in \mathbb{T}^{N}$, there exists a sequence $\alpha_{n} \rightarrow 0$ such that

$$
\begin{array}{lr}
\lim _{n \rightarrow \infty} \alpha_{n} w_{i}^{\alpha_{n}}(y)=\lambda_{i} & \text { for any } y \in \mathbb{T}^{N}, \\
\lim _{n \rightarrow \infty} w_{i}^{\alpha_{n}}(y)-w_{i}^{\alpha_{n}}\left(y_{0}\right)=v_{i}(y) & \text { uniformly in } \mathbb{T}^{N} .
\end{array}
$$

Moreover $v_{i}$ is Lipschitz continuous with constant $L_{R}$ and by standard stability result in viscosity solution theory $\left(\lambda_{i}, v_{i}\right)$ is a solution to (4.1). Finally it is possible to prove that the number $\lambda_{i}$ for which (4.1) admits a solution is univocally defined, while it is well known that in general the viscosity solution of (4.1) is not unique.

Definition 4.2. For any $i=1, \ldots, M$, the effective Hamiltonian $\bar{H}_{i}(x, r, p)$ associated to the Hamiltonian $H_{i}$ is defined by setting

$$
\bar{H}_{i}(x, r, p)=\lambda_{i}
$$

where $\lambda_{i}$ is given by Proposition 4.1 .

We now deduce some properties of the effective Hamiltonians.

Proposition 4.3. Assume (2.2). For any $i=1, \ldots, M$, the effective Hamiltonian $\bar{H}_{i}$ satisfies:

(i) $\bar{H}_{i}$ is continuous in $(x, r, p)$ and, for all $R>0$, there exists a modulus of continuity $\omega_{R}$ such that, for all $x, x^{\prime} \in \mathbb{R}^{N}, r, r^{\prime} \in \mathbb{R}^{M}, p, p^{\prime} \in \mathbb{R}^{N}$ with $|r|+\left|r^{\prime}\right|+|p|+\left|p^{\prime}\right| \leq R$, we have

$$
\left|\bar{H}_{i}(x, r, p)-\bar{H}_{i}\left(x^{\prime}, r^{\prime}, p^{\prime}\right)\right| \leq \omega_{R}\left(\left|x-x^{\prime}\right|\right)+\omega_{R}\left(\left|r-r^{\prime}\right|+\left|p-p^{\prime}\right|\right) .
$$

(ii) $\bar{H}_{i}$ is coercive in $p$ (uniformly with respect to $(x, r)$ ).

(iii) If $H_{i}$ is convex in $p$, then $\bar{H}_{i}$ is convex in $p$.

(iv) If $H_{i}$ satisfies (2.3), then $\bar{H}_{i}$ satisfies (2.3).

Proof. We first prove (i). Let $R>0$ and $(x, r, p),(\alpha, s, q) \in \mathbb{R}^{N} \times \mathbb{R}^{M} \times \mathbb{R}^{N}$ such that $|r|+|s|+|p|+|q| \leq R$. Let $v, w$ two periodic functions which satisfy

$$
\begin{aligned}
H_{i}(x, y, r, p+D v(y)) & =\bar{H}_{i}(x, r, p) \quad y \in \mathbb{T}^{N}, \\
H_{i}(x+\alpha, y, r+s, p+q+D w(y)) & =\bar{H}_{i}(x+\alpha, r+s, p+q) \quad y \in \mathbb{T}^{N} .
\end{aligned}
$$

By periodicity of $v$ and $w$, for any $\varepsilon>0$, the supremum

$$
\sup _{z, y \in \mathbb{R}^{N}}\left\{v(z)-w(y)-\frac{|z-y|^{2}}{\varepsilon^{2}}\right\}
$$


is achieved at some point $(\bar{z}, \bar{y})$ (which depends on $x, \alpha, p, q, r, s, \varepsilon)$. Moreover it is easy to see that, since $v, w$ are bounded Lipschitz continuous, we have

$$
\frac{|\bar{z}-\bar{y}|}{\varepsilon^{2}} \leq|D v|_{\infty},|D w|_{\infty} \leq L_{R}
$$

where $L_{R}$ is given by Proposition 4.1. Since $v$ is a viscosity subsolution of (4.4) and $w$ is a supersolution of (4.5), we obtain

$$
\begin{aligned}
H_{i}(x, \bar{z}, r, p+\bar{p}) & \leq \bar{H}_{i}(x, r, p), \\
H_{i}(x+\alpha, \bar{y}, r+s, p+q+\bar{p}) & \geq \bar{H}_{i}(x+\alpha, r+s, p+q) .
\end{aligned}
$$

By $(2.2)$ we get

$$
\begin{aligned}
\bar{H}_{i}(x+\alpha, r+s, p+q)-\bar{H}_{i}(x, r, p) \leq & H_{i}(x+\alpha, \bar{y}, r+s, p+q+\bar{p})-H_{i}(x, \bar{z}, r, p+\bar{p}) \\
= & H_{i}(x+\alpha, \bar{y}, r+s, p+q+\bar{p})-H_{i}(x, \bar{z}, r+s, p+q+\bar{p}) \\
& +H_{i}(x, \bar{z}, r+s, p+q+\bar{p})-H_{i}(x, \bar{z}, r, p+\bar{p}) \\
\leq & \omega\left(\left(1+R+L_{R}\right)(|\alpha|+|\bar{z}-\bar{y}|)\right)+\tilde{\omega}_{R}(|s|+|q|),
\end{aligned}
$$

where $\omega$ is given by (2.2) (iv) and $\tilde{\omega}_{R}$ is a modulus of continuity of the continuous function $H_{i}$ on the subset $\mathbb{R}^{N} \times \mathbb{T}^{N} \times[-R, R]^{M} \times B_{N}\left(0, R+L_{R}\right)$ given by (2.2) (iii). Sending $\varepsilon$ to 0 and setting $\omega_{R}(l)=\max \{\omega((1+R+$ $\left.\left.\left.L_{R}\right) l\right), \tilde{\omega}_{R}(l)\right\}$, we get

$$
\bar{H}_{i}(x+\alpha, r+s, p+q)-\bar{H}_{i}(x, r, p) \leq \omega_{R}(|\alpha|)+\omega_{R}(|s|+|q|),
$$

which ends the proof of (i).

The proof of (ii) and (iii) are standard, see [8].

We now prove that $\bar{H}_{i}, i=1, \ldots, M$, satisfies the monotonicity condition (2.3). We assume by contradiction that there exist $r, s \in \mathbb{R}^{M}$ for which $r_{j}-s_{j}=\max _{k=1, \ldots, M}\left\{r_{k}-s_{k}\right\} \geq 0$ and

$$
\bar{H}_{j}(x, r, p)<\bar{H}_{j}(x, s, p)
$$

for some $x, p \in \mathbb{R}^{N}$. Let $v, w$ be two periodic functions such that

$$
\begin{aligned}
H_{j}(x, y, r, p+D v) & =\bar{H}_{j}(x, r, p) & & y \in \mathbb{T}^{N}, \\
H_{j}(x, y, s, p+D w) & =\bar{H}_{j}(x, s, p) & & y \in \mathbb{T}^{N} .
\end{aligned}
$$

Since $v, w$ are bounded, by adding a constant we can assume w.l.o.g. $v>w$ in $\mathbb{T}^{N}$. By $(2.3)$

$$
H_{j}(x, y, r, p+D v)=\bar{H}_{j}(x, r, p)<\bar{H}_{j}(x, s, p) \leq H_{j}(x, y, s, p+D w) \leq H_{j}(x, y, r, p+D w)
$$

and for $\alpha$ sufficiently small

$$
\alpha v+H_{j}(x, y, r, p+D v)>\alpha w+H_{j}(x, y, r, p+D w) \quad y \in \mathbb{T}^{N} .
$$

This last inequality gives a contradiction by the comparison principle for (4.2). 


\section{The Homogenization THEOREM}

In this section we prove the homogenization theorem for the problem (1.1).

Proposition 5.1. Assume (2.2), (2.3) and (2.4). Then there exists a unique solution $u \in B U C\left(\mathbb{R}^{N} \times[0, T]\right)$ of

$$
\begin{cases}\frac{\partial u_{i}}{\partial t}+\bar{H}_{i}\left(x, u, D u_{i}\right)=0 & (x, t) \in \mathbb{R}^{N} \times(0, \infty), \\ u_{i}(x, 0)=u_{0, i}(x) & x \in \mathbb{R}^{N}, \quad i=1, \ldots, M .\end{cases}
$$

Proof. The difficulty here is that the comparison principle for the limit system (5.1) is not a straightforward consequence of Proposition 4.3. Indeed, the regularity of the Hamiltonians $\bar{H}_{i}$ is weaker than (2.2) (in particular compare (2.2) (iv) and (4.3)). To prove the comparison principle we first prove comparison in the case where either the subsolution or the supersolution is bounded Lipschitz continuous and then we proceed by approximation.

Suppose that $u_{0}$ is bounded Lipschitz continuous, $u$ is a bounded subsolution and $v$ a bounded supersolution of (5.1) and $u$, for instance, is Lipschitz continuous (with constant $L$ ). Arguing as in Proposition 3.1 and looking carefully at the proof of Proposition 4.3 (see in particular (4.6)), it follows that the second estimate in (3.9) could be replaced by

$$
|\bar{p}| \leq L,
$$

and therefore, setting $R=|v|_{\infty}+2 M+L$, from (4.3), (3.10) becomes

$$
\mathcal{T}_{1} \leq \omega_{R}(L \alpha)
$$

The term $\mathcal{T}_{2}$ does not exist since $\bar{H}_{\bar{j}}$ does not depend on $t$. We deal with $\mathcal{T}_{3}$ using again (4.3) and $\mathcal{T}_{4} \leq 0$ as in the proof of Proposition 4.3. The rest of the proof is the same (even easier since $u(\cdot, 0)$ is Lipschitz continuous). Hence we have comparison between Lipschitz continuous sub- and supersolutions. In particular, by Perron's method, for any Lipschitz continuous $u_{0}$, there exists a unique Lipschitz continuous solution $u$ of (5.1). Moreover, repeating the beginning of the proof of Proposition 3.3, we obtain (3.25), i.e.

$$
\max _{1 \leq j \leq M} \sup _{\mathbb{R}^{N} \times[0, T]} u_{j}-v_{j} \leq \max _{1 \leq j \leq M} \sup _{\mathbb{R}^{N}}\left(u_{j}(\cdot, 0)-v_{j}(\cdot, 0)\right)^{+}
$$

if $u$ is a subsolution and $v$ a supersolution of (5.1) and either $u$ or $v$ is Lipschitz continuous.

Now, consider the case when $u_{0}$ is $B U C$. Let $u$ (respectively $v$ ) be a $B U C$ subsolution (respectively supersolution) of (5.1). For all $\gamma>0$, there exists a Lipschitz continuous function $u_{0}^{\gamma}$ such that

$$
u_{0}^{\gamma} \leq u_{0} \leq u_{0}^{\gamma}+\gamma \quad \text { in } \mathbb{R}^{N} .
$$

Let $u^{\gamma}$ (respectively $v^{\gamma}$ ) be the Lipschitz continuous solution to (5.1) with initial data $u_{0}^{\gamma}$ (respectively $u_{0}^{\gamma}+\gamma$ ). By comparison in the Lipschitz case, $u^{\gamma} \leq v^{\gamma}$. From (5.2) and (5.3), it follows

$$
u \leq u^{\gamma}+\gamma \leq v^{\gamma}+\gamma \leq v+2 \gamma \quad \text { in } \mathbb{R}^{N} \times[0, T] .
$$

Since the previous inequality is true for all $\gamma>0$, we obtain the desired comparison $u \leq v$. We also obtain the existence and the uniqueness of a $B U C$ solution as a byproduct of this latter proof.

Theorem 5.2. Assume (2.2), (2.3) and (2.4). The viscosity solution $u^{\varepsilon}$ of (1.1) converges locally uniformly on $\mathbb{R}^{N} \times[0, T]$ to the viscosity solution $u \in B U C\left(\mathbb{R}^{N} \times[0, T]\right)$ of $(5.1)$. 
Proof. By Proposition 3.3 there exists a continuous solution $u^{\varepsilon}$ of (1.1) which is bounded independently of $\varepsilon$. It follows that we can define the half-relaxed limits

$$
\bar{u}(x, t)=\limsup _{\varepsilon \rightarrow 0,\left(x_{\varepsilon}, t_{\varepsilon}\right) \rightarrow(x, t)} u^{\varepsilon}\left(x_{\varepsilon}, t_{\varepsilon}\right) \quad \text { and } \quad \underline{u}(x, t)=\liminf _{\varepsilon \rightarrow 0,\left(x_{\varepsilon}, t_{\varepsilon}\right) \rightarrow(x, t)} u^{\varepsilon}\left(x_{\varepsilon}, t_{\varepsilon}\right) .
$$

Let us mention at this step that we could use Ascoli's theorem in view of the equicontinuity property of Proposition 3.3 (ii) to obtain a limit for $u^{\varepsilon}$ along a subsequence. We choose to use the half-relaxed limits since it is not much more complicated and it does not require uniform moduli of continuity for the $u^{\varepsilon}$ 's.

We first show that $\bar{u}$ is a viscosity subsolution of the system (5.1). We assume there exist $j \in\{1, \ldots, M\}$ and $\phi \in C^{1}$ such that $\bar{u}_{j}-\phi$ has a strict maximum point at some $(\bar{x}, \bar{t})$ with $\bar{t}>0$ and $\bar{u}_{j}(\bar{x}, \bar{t})=\phi(\bar{x}, \bar{t})$. We assume w.l.o.g. $j=1$. By Proposition 4.1, there exists a corrector $v$ for $(\bar{x}, \bar{u}(\bar{x}, \bar{t}), D \phi(\bar{x}, \bar{t}))$, i.e. a viscosity solution of

$$
H_{1}(\bar{x}, y, \bar{u}(\bar{x}, \bar{t}), D \phi(\bar{x}, \bar{t})+D v(y))=\bar{H}_{1}(\bar{x}, \bar{u}(\bar{x}, \bar{t}), D \phi(\bar{x}, \bar{t})) \quad y \in \mathbb{T}^{N}
$$

Define the "perturbed test-function"

$$
\phi^{\varepsilon, \alpha}(x, y, t)=\phi(x, t)+\varepsilon v\left(\frac{y}{\varepsilon}\right)+\frac{|x-y|^{2}}{\alpha^{2}} .
$$

By classical results on viscosity solutions (see [3], Lem. 4.3, or [2]), we have, since $\bar{u}_{1}-\phi$ has a strict maximum point at $(\bar{x}, \bar{t})$, up to extract subsequences, there exist $\left(x_{\varepsilon, \alpha}, y_{\varepsilon, \alpha}, t_{\varepsilon, \alpha}\right) \in \mathbb{R}^{N} \times \mathbb{R}^{N} \times(0, T]$ and $\left(x_{\varepsilon}, t_{\varepsilon}\right) \in$ $\mathbb{R}^{N} \times(0, T]$ such that $\left(x_{\varepsilon, \alpha}, y_{\varepsilon, \alpha}, t_{\varepsilon, \alpha}\right)$ is a local maximum of $u_{1}^{\varepsilon}(x, t)-\phi^{\varepsilon, \alpha}(x, y, t)$ and

$$
\begin{aligned}
& \left(x_{\varepsilon, \alpha}, y_{\varepsilon, \alpha}, t_{\varepsilon, \alpha}\right) \rightarrow\left(x_{\varepsilon}, x_{\varepsilon}, t_{\varepsilon}\right) \quad \text { as } \alpha \rightarrow 0, \\
& \left(x_{\varepsilon}, t_{\varepsilon}\right) \rightarrow(\bar{x}, \bar{t}) \quad \text { as } \varepsilon \rightarrow 0, \\
& \lim _{\varepsilon \rightarrow 0} \lim _{\alpha \rightarrow 0} u_{1}^{\varepsilon}\left(x_{\varepsilon, \alpha}, t_{\varepsilon, \alpha}\right)=u_{1}(\bar{x}, \bar{t}) .
\end{aligned}
$$

Since $u_{1}^{\varepsilon}(x, t)-\phi^{\varepsilon, \alpha}\left(x, y_{\varepsilon, \alpha}, t\right)$ has a maximum point at $\left(x_{\varepsilon, \alpha}, t_{\varepsilon, \alpha}\right)$ and $u^{\varepsilon}$ is a subsolution of (1.1), setting $p_{\varepsilon, \alpha}=2 \frac{x_{\varepsilon, \alpha}-y_{\varepsilon, \alpha}}{\alpha^{2}}$, we get

$$
\phi_{t}\left(x_{\varepsilon, \alpha}, t_{\varepsilon, \alpha}\right)+H_{1}\left(x_{\varepsilon, \alpha}, \frac{x_{\varepsilon, \alpha}}{\varepsilon}, u^{\varepsilon}\left(x_{\varepsilon, \alpha}, t_{\varepsilon, \alpha}\right), D \phi\left(x_{\varepsilon, \alpha}, t_{\varepsilon, \alpha}\right)+p_{\varepsilon, \alpha}\right) \leq 0 .
$$

Since $v$ is a supersolution of (5.4) and $y \mapsto v(y)-\psi^{\varepsilon, \alpha}(\varepsilon y)$ has a minimum point at $y_{\varepsilon, \alpha} / \varepsilon$ with

$$
\psi^{\varepsilon, \alpha}(y)=-\frac{1}{\varepsilon}\left(\frac{\left|x_{\varepsilon, \alpha}-y\right|^{2}}{\alpha^{2}}+\phi\left(x_{\varepsilon, \alpha}, t_{\varepsilon, \alpha}\right)-u_{1}^{\varepsilon}\left(x_{\varepsilon, \alpha}, t_{\varepsilon, \alpha}\right)\right),
$$

we get

$$
H_{1}\left(\bar{x}, \frac{y_{\varepsilon, \alpha}}{\varepsilon}, \bar{u}(\bar{x}, \bar{t}), D \phi(\bar{x}, \bar{t})+p_{\varepsilon, \alpha}\right) \geq \bar{H}_{1}(\bar{x}, \bar{u}(\bar{x}, \bar{t}), D \phi(\bar{x}, \bar{t}))
$$

The corrector $v$ is Lipschitz continuous by coercivity of $H_{1}$ (see Prop. 4.1). Therefore, from (4.6), we have

$$
\left|p_{\varepsilon, \alpha}\right| \leq|D v|_{\infty} \leq L_{R} \quad \text { with } R=|D \phi(\bar{x}, \bar{t})|+|\bar{u}|_{\infty}
$$

By (5.6) and (5.7), we have

$$
\begin{aligned}
\phi_{t}(\bar{x}, \bar{t})+\bar{H}_{1}(\bar{x}, \bar{u}(\bar{x}, \bar{t}), D \phi(\bar{x}, \bar{t})) \leq & \phi_{t}(\bar{x}, \bar{t})-\phi_{t}\left(x_{\varepsilon, \alpha}, t_{\varepsilon, \alpha}\right)+H_{1}\left(\bar{x}, \frac{y_{\varepsilon, \alpha}}{\varepsilon}, \bar{u}(\bar{x}, \bar{t}), D \phi(\bar{x}, \bar{t})+p_{\varepsilon, \alpha}\right) \\
& -H_{1}\left(x_{\varepsilon, \alpha}, \frac{x_{\varepsilon, \alpha}}{\varepsilon}, u^{\varepsilon}\left(x_{\varepsilon, \alpha}, t_{\varepsilon, \alpha}\right), D \phi\left(x_{\varepsilon, \alpha}, t_{\varepsilon, \alpha}\right)+p_{\varepsilon, \alpha}\right) .
\end{aligned}
$$


Since $\left(x_{\varepsilon, \alpha}, t_{\varepsilon, \alpha}\right) \rightarrow(\bar{x}, \bar{t})$ and $\phi$ is smooth we get

$$
\lim _{\varepsilon \rightarrow 0} \lim _{\alpha \rightarrow 0} \phi_{t}(\bar{x}, \bar{t})-\phi_{t}\left(x_{\varepsilon, \alpha}, t_{\varepsilon, \alpha}\right)=0 .
$$

To estimate the second term of the right-hand side we set

$$
\begin{aligned}
& \mathcal{T}_{1}=H_{1}\left(\bar{x}, \frac{y_{\varepsilon, \alpha}}{\varepsilon}, \bar{u}(\bar{x}, \bar{t}), D \phi(\bar{x}, \bar{t})+p_{\varepsilon, \alpha}\right)-H_{1}\left(x_{\varepsilon, \alpha}, \frac{x_{\varepsilon, \alpha}}{\varepsilon}, \bar{u}(\bar{x}, \bar{t}), D \phi(\bar{x}, \bar{t})+p_{\varepsilon, \alpha}\right), \\
& \mathcal{T}_{2}=H_{1}\left(x_{\varepsilon, \alpha}, \frac{x_{\varepsilon, \alpha}}{\varepsilon}, \bar{u}(\bar{x}, \bar{t}), D \phi(\bar{x}, \bar{t})+p_{\varepsilon, \alpha}\right)-H_{1}\left(x_{\varepsilon, \alpha}, \frac{x_{\varepsilon, \alpha}}{\varepsilon}, u^{\varepsilon}\left(x_{\varepsilon, \alpha}, t_{\varepsilon, \alpha}\right), D \phi(\bar{x}, \bar{t})+p_{\varepsilon, \alpha}\right), \\
& \mathcal{T}_{3}=H_{1}\left(x_{\varepsilon, \alpha}, \frac{x_{\varepsilon, \alpha}}{\varepsilon}, u^{\varepsilon}\left(x_{\varepsilon, \alpha}, t_{\varepsilon, \alpha}\right), D \phi(\bar{x}, \bar{t})+p_{\varepsilon, \alpha}\right)-H_{1}\left(x_{\varepsilon, \alpha}, \frac{x_{\varepsilon, \alpha}}{\varepsilon}, u^{\varepsilon}\left(x_{\varepsilon, \alpha}, t_{\varepsilon, \alpha}\right), D \phi\left(x_{\varepsilon, \alpha}, t_{\varepsilon, \alpha}\right)+p_{\varepsilon, \alpha}\right) .
\end{aligned}
$$

From (2.2) (iv) and (5.8),

$$
\begin{aligned}
\mathcal{T}_{1} & \leq \omega\left(\left(1+\left|D \phi(\bar{x}, \bar{t})+p_{\varepsilon, \alpha}\right|\right)\left(\left|\bar{x}-x_{\varepsilon, \alpha}\right|+\frac{\left|x_{\varepsilon, \alpha}-y_{\varepsilon, \alpha}\right|}{\varepsilon}\right)\right) \\
& \leq \omega\left(\left(1+R+L_{R}\right)\left(\left|\bar{x}-x_{\varepsilon, \alpha}\right|+\frac{L_{R} \alpha^{2}}{\varepsilon}\right)\right)
\end{aligned}
$$

and therefore

$$
\lim _{\varepsilon \rightarrow 0} \lim _{\alpha \rightarrow 0} \mathcal{T}_{1}=0 .
$$

To deal with $\mathcal{T}_{2}$, we use the monotonicity assumption (2.3). Let $\delta>0$. At first, up to extract some subsequences, we can assume by definition of $\bar{u}$ that for $\alpha, \varepsilon$ small enough with $\alpha \ll \varepsilon$, we have

$$
u_{j}^{\varepsilon}\left(x_{\varepsilon, \alpha}, t_{\varepsilon, \alpha}\right)-\bar{u}_{j}(\bar{x}, \bar{t}) \leq \frac{\delta}{2} \quad \text { for } 2 \leq j \leq M .
$$

Hence

$$
\max \left\{\bar{u}_{1}(\bar{x}, \bar{t})+\delta-\bar{u}_{1}(\bar{x}, \bar{t}), u_{2}^{\varepsilon}\left(x_{\varepsilon, \alpha}, t_{\varepsilon, \alpha}\right)-\bar{u}_{2}(\bar{x}, \bar{t}), \ldots, u_{M}^{\varepsilon}\left(x_{\varepsilon, \alpha}, t_{\varepsilon, \alpha}\right)-\bar{u}_{M}(\bar{x}, \bar{t})\right\}=\delta
$$

is achieved for the first component. Set $r_{\delta}=\left(\bar{u}_{1}(\bar{x}, \bar{t})+\delta, u_{2}^{\varepsilon}\left(x_{\varepsilon, \alpha}, t_{\varepsilon, \alpha}\right), \ldots, u_{M}^{\varepsilon}\left(x_{\varepsilon, \alpha}, t_{\varepsilon, \alpha}\right)\right)$, then by $(2.3)$

$$
H_{1}\left(x_{\varepsilon, \alpha}, \frac{x_{\varepsilon, \alpha}}{\varepsilon}, \bar{u}(\bar{x}, \bar{t}), D \phi(\bar{x}, \bar{t})+p_{\varepsilon, \alpha}\right)-H_{1}\left(x_{\varepsilon, \alpha}, \frac{x_{\varepsilon, \alpha}}{\varepsilon}, r_{\delta}, D \phi(\bar{x}, \bar{t})+p_{\varepsilon, \alpha}\right) \leq 0 .
$$

Then

$$
\mathcal{T}_{2} \leq H_{1}\left(x_{\varepsilon, \alpha}, \frac{x_{\varepsilon, \alpha}}{\varepsilon}, r_{\delta}, D \phi(\bar{x}, \bar{t})+p_{\varepsilon, \alpha}\right)-H_{1}\left(x_{\varepsilon, \alpha}, \frac{x_{\varepsilon, \alpha}}{\varepsilon}, u^{\varepsilon}\left(x_{\varepsilon, \alpha}, t_{\varepsilon, \alpha}\right), D \phi(\bar{x}, \bar{t})+p_{\varepsilon, \alpha}\right):=\mathcal{T}_{4} .
$$

To prove

$$
\lim _{\varepsilon \rightarrow 0} \lim _{\alpha \rightarrow 0} \mathcal{T}_{3}=0 \quad \text { and } \quad \lim _{\delta \rightarrow 0} \lim _{\varepsilon \rightarrow 0} \lim _{\alpha \rightarrow 0} \mathcal{T}_{4}=0,
$$

we use the uniform continuity of $H_{1}$ on compact subsets. We have

$$
\lim _{\varepsilon \rightarrow 0} \lim _{\alpha \rightarrow 0}\left(x_{\varepsilon, \alpha}, u^{\varepsilon}\left(x_{\varepsilon, \alpha}, t_{\varepsilon, \alpha}\right), D \phi\left(x_{\varepsilon, \alpha}, t_{\varepsilon, \alpha}\right)\right)=(\bar{x}, \bar{u}(\bar{x}, \bar{t}), D \phi(\bar{x}, \bar{t}))
$$


and

$$
\lim _{\delta \rightarrow 0} \lim _{\varepsilon \rightarrow 0} \lim _{\alpha \rightarrow 0} r_{\delta}=\bar{u}(\bar{x}, \bar{t})
$$

Since $x_{\varepsilon, \alpha}, y_{\varepsilon, \alpha} \rightarrow \bar{x}$ we have $x_{\varepsilon, \alpha}, y_{\varepsilon, \alpha}$ stay in some ball $\bar{B}(\bar{x}, \bar{R})$. Hence choosing $K=\bar{B}(\bar{x}, \bar{R}) \times \mathbb{T}^{N}$ $\times\left[-|\bar{u}|_{\infty}-1,|\bar{u}|_{\infty}+1\right]^{M} \times \bar{B}\left(0,|D \phi(\bar{x}, \bar{t})|+L_{R}+1\right)$, by uniform continuity of $H_{1}$ on $K$, (5.11) holds. The periodicity of $H_{1}$ allows to deal with $x_{\varepsilon, \alpha} / \varepsilon$ which is not bounded.

Finally, sending $\alpha \rightarrow 0$ at first, then $\varepsilon \rightarrow 0$ and finally $\delta \rightarrow 0$, we conclude that the right-hand side of (5.9) is non positive. Then $\bar{u}$ is a subsolution of (5.1).

We prove that $\underline{u}$ is a viscosity supersolution of (5.1) in a similar way. From Proposition 3.1, we then obtain $\bar{u} \leq \underline{u}$ in $\mathbb{R}^{N} \times[0, T]$. It follows that $\bar{u}=\underline{u}:=u$ where $u$ is the (local) uniform limit of the $u^{\varepsilon}$ 's.

\section{Remark 5.3.}

(1) As mentioned above, the coercivity of the Hamiltonians plays a crucial role: it ensures the Lipschitz continuity of the correctors which allows us to deal with $\mathcal{T}_{3}$ and $\mathcal{T}_{4}$ with weak regularity assumptions with respect to $(r, p)$ in $(2.2)$. When the Hamiltonians are not coercive anymore (and therefore the corrector is not necessarily Lipschitz continuous), the proof is more delicate. A way to solve this problem is to use the ideas of Barles [4] and his " $F^{k}$-trick" (see [4], Lem. 2.1 and Thm. 2.1).

(2) In the Lipschitz case (when $u_{0}$ is Lipschitz continuous), the above proof can be done in a simpler way using the uniform Lipschitz estimates on $u^{\varepsilon}$ given by Proposition 3.3.

\section{EXAMPLE}

We first describes a class of systems (2.1) which satisfy (2.3). We assume that the Hamiltonians $H_{j}$ satisfy the following assumption (see [10])

$$
\begin{aligned}
& \text { There exists } c_{i j} \in \mathbb{R}, 1 \leq i, j \leq M \text {, s.t. } \sum_{j=1}^{M} c_{j i} \geq 0 \text { and } \\
& \text { for any }(x, y, r, p) \in \mathbb{R}^{N} \times \mathbb{R}^{N} \times \mathbb{R}^{M} \times \mathbb{R}^{N}, \delta>0, \\
& c_{j i} \delta \leq H_{i}\left(x, y, r+\delta e_{j}, p\right)-H_{i}(x, y, r, p) \leq 0 \quad \text { if } j \neq i, \\
& c_{i i} \delta \leq H_{i}\left(x, y, r+\delta e_{i}, p\right)-H_{i}(x, y, r, p)
\end{aligned}
$$

where $\left(e_{1}, \ldots, e_{n}\right)$ is the canonical basis of $\mathbb{R}^{M}$. Note that necessarily $c_{j i} \leq 0$ for $i \neq j$ and $c_{i i} \geq 0$.

In the next proposition we prove that the assumption (6.1) implies the monotonicity condition (2.3).

Proposition 6.1. Condition (6.1) implies (2.3).

Proof. Assume that $r_{j}-s_{j}=\max _{k=1, \ldots, M}\left\{r_{k}-s_{k}\right\} \geq 0$. For simplicity, we drop the dependence in $(x, y, p)$ in $H(x, y, r, p)$ in the proof of the proposition since these variables do not play any role here. We have

$$
\begin{aligned}
H_{j}(r)-H_{j}(s)= & H_{j}\left(r_{1}, r_{2}, \ldots, r_{j}, \ldots, r_{M}\right)-H_{j}\left(s_{1}, s_{2}, \ldots, s_{j}, \ldots, s_{M}\right) \\
= & H_{j}\left(r_{1}, r_{2}, \ldots, r_{j}, \ldots, r_{M}\right)-H_{j}\left(s_{1}, r_{2}, \ldots, r_{j}, \ldots, r_{M}\right) \\
& +H_{j}\left(s_{1}, r_{2}, \ldots, r_{j}, \ldots, r_{M}\right)-H_{j}\left(s_{1}, s_{2}, r_{3}, \ldots, r_{j}, \ldots, r_{M}\right) \\
& +\ldots \\
& +H_{j}\left(s_{1}, \ldots, s_{j-1}, r_{j}, \ldots, r_{M}\right)-H_{j}\left(s_{1}, \ldots, s_{j}, r_{j+1}, \ldots, r_{M}\right) \\
& +\ldots \\
& +H_{j}\left(s_{1}, \ldots, s_{M-1}, r_{M}\right)-H_{j}\left(s_{1}, \ldots, s_{M}\right) .
\end{aligned}
$$


If $k \neq j$,

$$
\begin{aligned}
& H_{j}\left(s_{1}, \ldots s_{k-1}, r_{k}, r_{k+1}, \ldots, r_{M}\right)-H_{j}\left(s_{1}, \ldots, s_{k-1}, s_{k}, r_{k+1}, \ldots, r_{M}\right) \geq \\
& \left\{\begin{array}{cl}
0 & \text { if } r_{k}-s_{k}<0 \\
c_{k j}\left(r_{k}-s_{k}\right) & \text { if } r_{k}-s_{k} \geq 0
\end{array} \geq c_{k j}\left(r_{j}-s_{j}\right)\right.
\end{aligned}
$$

since $c_{k j} \leq 0$ and $r_{j}-s_{j}=\max _{k=1, \ldots, M}\left\{r_{k}-s_{k}\right\} \geq 0$. Moreover,

$$
H_{j}\left(s_{1}, \ldots, s_{j-1}, r_{j}, r_{j+1}, \ldots, r_{M}\right)-H_{j}\left(s_{1}, \ldots, s_{j-1}, s_{j}, r_{j+1}, \ldots, r_{M}\right) \geq c_{j j}\left(r_{j}-s_{j}\right) .
$$

It follows

$$
H_{j}(r)-H_{j}(s) \geq \sum_{k=1}^{M} c_{k j}\left(r_{j}-s_{j}\right) \geq 0
$$

as desired.

Remark 6.2. Property (2.3) is not equivalent to (6.1). More precisely, if (2.3) holds, the existence of $c_{j i}$ for $j \neq i$ is not always true (the others assertions hold). Indeed, for $M=2$, consider for instance $H_{1}\left(r_{1}, r_{2}\right)=$ $\mathrm{e}^{r_{1}-r_{2}}+2 r_{1}-r_{2}$ (and define $H_{2}$ symmetrically). Then

$$
H_{1}\left(r_{1}+\delta, r_{2}+\mu\right)-H_{1}\left(r_{1}, r_{2}\right)=\mathrm{e}^{r_{1}-r_{2}}\left(\mathrm{e}^{\delta-\mu}-1\right)+2 \delta-\mu \geq \delta
$$

when $\mu \leq \delta$. This ensures (2.3) with $\lambda_{0}=1$. Nevertheless, $H_{1}\left(r_{1}, r_{2}+\mu\right)-H_{1}\left(r_{1}, r_{2}\right) \sim-\left(1+\mathrm{e}^{r_{1}-r_{2}}\right) \mu$ for small $\mu$ and $1+\mathrm{e}^{r_{1}-r_{2}}$ is not bounded.

A particular case of monotone systems are the weakly coupled systems (2.6). For (2.6), assumption (6.1) is satisfied if

$$
c_{i i}(x, y) \geq 0, \quad c_{j i}(x, y) \leq 0 \text { for } j \neq i \quad \text { and } \sum_{j=1}^{M} c_{j i}(x, y) \geq 0
$$

for any $x, y \in \mathbb{R}^{N}, i, j \in\{1, \ldots, M\}$. Let us consider a specific example of weakly coupled system for which it is possible to have an explicit formula for the effective Hamiltonians. Consider the system

$$
\frac{\partial u_{i}}{\partial t}+\left|\frac{\partial u_{i}}{\partial x}\right|+\sum_{j=1}^{M} c_{j i}\left(\frac{x}{\varepsilon}\right) u_{j}=0 \quad(x, t) \in \mathbb{R} \times[0, \infty),
$$

where the $c_{j i}$ 's satisfy $(6.2)$. The associated cell problems are

$$
\left|p+v^{\prime}(y)\right|+\sum_{j=1}^{M} c_{j i}(y) r_{j}=\lambda \quad y \in[0,1], \lambda \in \mathbb{R},
$$

for $i=1, \ldots, M$. We rewrite $(6.3)$ as

$$
\left|p+v^{\prime}(y)\right|=\lambda+f(y) \quad y \in[0,1]
$$


where $f(y)=-\sum_{j=1}^{M} c_{j i}(y) r_{j}$. The effective Hamiltonian for (6.4) is given by (see [8])

$$
\bar{H}(p)=\max \left\{-\min _{[0,1]} f,|p|-\int_{0}^{1} f(y) \mathrm{d} y\right\}
$$

and therefore we get the effective Hamiltonian for (6.3)

$$
\bar{H}_{i}(r, p)=\max \left\{\max _{[0,1]} \sum_{j=1}^{M} c_{j i}(y) r_{j},|p|+\sum_{j=1}^{M} r_{j} \int_{0}^{1} c_{j i}(y) \mathrm{d} y\right\} .
$$

A natural question is that if the problem (5.1) which arises in the homogenized limit of the weakly coupled system (2.6) is still of weakly coupled type. Whereas the answer is positive if the coefficients $c_{i j}$ are independent of $y$, in general it is not necessarily true.

Proposition 6.3. Assume that (2.2) holds and that the coefficients $c_{i j}$ in (2.6) are constant and satisfy (6.2). Then

$$
\bar{H}_{i}(r, p)=\bar{H}_{i}(p)+\sum_{j=1}^{M} c_{j i} r_{j}
$$

where $\bar{H}_{i}(p)$ is the effective Hamiltonian of $H_{i}(x, p)$, i.e. the unique $\lambda \in \mathbb{R}$ for which the equation

$$
H_{i}(y, p+D v(y))=\bar{H}_{i}(p) \quad y \in \mathbb{T}^{N}
$$

admits a viscosity solution.

Proof. By definition, there exists a viscosity solution to

$$
H_{i}(y, p+D v(y))+\sum_{j=1}^{M} c_{j i} r_{j}=\bar{H}_{i}(r, p), \quad y \in \mathbb{T}^{N}
$$

or equivalently to

$$
H_{i}(y, p+D v(y))=\bar{H}_{i}(r, p)-\sum_{j=1}^{M} c_{j i} r_{j} \quad y \in \mathbb{T}^{N} .
$$

By (6.8) and the uniqueness of the effective Hamiltonian, the constant in the right hand side of the previous equation is given by $\bar{H}_{i}(p)$, hence the formula (6.7).

The following example shows that if the coupling coefficients $c_{i j}$ are not constants, the limit system is not necessarily weakly coupled. Consider the 1 -dimensional case (6.3). Take $i=1$ and $r=\left(r_{1}, 0, \ldots, 0\right)$, then

$$
\bar{H}_{1}(r, p)=\max \left\{\max _{[0,1]} c_{11}(y) r_{1},|p|+r_{1} \int_{0}^{1} c_{11}(y) \mathrm{d} y\right\} .
$$

If $\max _{[0,1]} c_{11}=\alpha, \min _{[0,1]} c_{11}=\beta$ and $\int_{0}^{1} c_{11}(y) \mathrm{d} y=\gamma$, with $\alpha>\gamma>\beta \geq 0$, then for $p \neq 0$ fixed

$$
\bar{H}_{1}(r, p)= \begin{cases}\beta r_{1} & \text { if } r_{1} \leq|p| /(\beta-\gamma), \\ \gamma r_{1}+|p| & \text { if }|p| /(\beta-\gamma) \leq r_{1} \leq|p| /(\alpha-\gamma), \\ \alpha r_{1} & \text { if } r_{1} \geq|p| /(\alpha-\gamma) .\end{cases}
$$


Then $H_{1}(r, p)$ is not a linear function of $r$ and therefore is not of weakly coupled type.

By the formula (6.6) it is possible to see another typical phenomenon in homogenization of Hamilton-Jacobi equation, the presence of a flat part in the graph of effective Hamiltonian (see $[8,16]$ ).

Acknowledgements. This work was initiated while the second author was invited at the Dipartimento di Metodi e Modelli Matematici per le Scienze Applicate of the University "La Sapienza" (Roma). He would like to thank the department for hospitality.

\section{REFERENCES}

[1] O. Alvarez and M. Bardi, Singular perturbations of nonlinear degenerate parabolic PDEs: a general convergence result. Arch. Ration. Mech. Anal. 170 (2003) 17-61.

[2] M. Bardi and I. Capuzzo-Dolcetta, Optimal control and viscosity solutions of Hamilton-Jacobi-Bellman equations. Birkhäuser Boston Inc., Boston, MA, USA (1997).

[3] G. Barles, Solutions de viscosité des équations de Hamilton-Jacobi. Springer-Verlag, Paris, France (1994).

[4] G. Barles, Some homogenization results for non-coercive Hamilton-Jacobi equations. Calc. Var. Partial Differential Equations 30 (2007) 449-466.

[5] G. Barles, S. Biton and O. Ley, A geometrical approach to the study of unbounded solutions of quasilinear parabolic equations. Arch. Ration. Mech. Anal. 162 (2002) 287-325.

[6] F. Camilli and P. Loreti, Comparison results for a class of weakly coupled systems of eikonal equations. Hokkaido Math. J. 37 (2008) 349-362.

[7] I. Capuzzo-Dolcetta and H. Ishii, On the rate of convergence in homogenization of Hamilton-Jacobi equations. Indiana Univ. Math. J. 50 (2001) 1113-1129.

[8] M.C. Concordel, Periodic homogenization of Hamilton-Jacobi equations: additive eigenvalues and variational formula. Indiana Univ. Math. J. 45 (1996) 1095-1117.

[9] A. Eizenberg and M. Freidlin, On the Dirichlet problem for a class of second order PDE systems with small parameter. Stochastics Stochastics Rep. 33 (1990) 111-148.

[10] H. Engler and S.M. Lenhart, Viscosity solutions for weakly coupled systems of Hamilton-Jacobi equations. Proc. London Math. Soc. (3) 63 (1991) 212-240.

[11] L.C. Evans, The perturbed test function method for viscosity solutions of nonlinear PDE. Proc. Roy. Soc. Edinburgh Sect. A 111 (1989) 359-375

[12] H. Ishii, Perron's method for monotone systems of second-order elliptic partial differential equations. Differential Integral Equations 5 (1992) 1-24.

[13] H. Ishii and S. Koike, Remarks on elliptic singular perturbation problems. Appl. Math. Optim. 23 (1991) 1-15.

[14] H. Ishii and S. Koike, Viscosity solutions for monotone systems of second-order elliptic PDEs. Comm. Partial Differential Equations 16 (1991) 1095-1128.

[15] P.-L. Lions and P.E. Souganidis, Correctors for the homogenization of Hamilton-Jacobi equations in the stationary ergodic setting. Comm. Pure Appl. Math. 56 (2003) 1501-1524.

[16] P.-L. Lions, B. Papanicolaou and S.R.S. Varadhan, Homogenization of Hamilton-Jacobi equations. Preprint (1986).

[17] K. Shimano, Homogenization and penalization of functional first-order PDE. NoDEA Nonlinear Differ. Equ. Appl. 13 (2006) $1-21$. 